\title{
Improvement in quality testing of Braille printer output with Euclidean distance measurement using camera calibration
}

\author{
Suman Karmakar*, Rudra Prasad Chatterjee* and Uma Dutta
}

\author{
Central Mechanical Engineering Research Institute,M.G. Avenue, Durgapur-713209, India \\ E-mails:(r_chatterjee@cmeri.res.in (Rudra Prasad Chatterjee*), suman2007@gmail.com (Suman Karmakar*) *Corresponding authors); \\ umadutta@cmeri.res.in (Uma Dutta)
}

\begin{abstract}
This paper focuses on quality testing of Braille printed paper using calibrated camera by detecting dots and measuring the Euclidean distances between them with equal gap, vertically and horizontally. For higher accuracy, camera calibration is essential to observe a planar checker board pattern from different distances and orientations. In this process, the position of the camera is fixed and the pattern can be freely moved. Radial lens distortion is modeled. Machine simulation and experimental results have also been discussed. Quality improvement can be achieved by giving a feedback after finding the distorted edges from image processing of the paper. This approach thus definitely helps the blind reader to avoid disturbances in reading the printed documents by both, single sided and inter-point printer.
\end{abstract}

Keywords: Image processing, camera calibration, quality testing of Braille printer

\section{Introduction}

Braille, a touch-reading system for the visually impaired people was first introduced in 1825 by Louis Braille (Hentzshel, 1993). Braille is a primary medium of reading and writing for the blind people or having low vision. Many blind and visually impaired individuals find their ability to access information more quickly and perform tasks that involve reading or writing more efficiently using Braille than by listening to a personal reader, dictating to personal secretary or alternative technologies such as audio recordings, talking computers, or other electronic devices. Braille character appears in dot format on Braille paper that can be identified by blind people on touching the enclave. To identify the proper position of the dots in the printer output, we need to maintain a fixed gap between every dot, as well as between every letter and every line. A method of thorough image processing to measure the gap between dots can help in this case. Generally, images taken by the digital camera result into a distorted image due to the lens distortion i.e. the actual output is always not available. Geometric distortion is an error on an image, between the actual image coordinates and the ideal image coordinates. Among various such nonlinear distortions, the radial distortion, which is along radial direction from the center of distortions, is the most severe part and is also the most common. Straight lines in the undistorted subject bulge in the characteristic barrel fashion, in the image. Straight lines running through the image center remain straight and a circle concentric with the image center remains circle, although its radius is affected. The most typical cases of distortion, barrel and pin-cushion distortion are primarily radial in nature, with a relatively simple one or two parameter model accounting for most of the distortion. Thus Camera calibration is a necessary step in 3-D computer vision in order to extract metric information from 2$\mathrm{D}$ images. The term, Camera re-sectioning (often called, camera calibration) is the process of finding the true parameters of the camera that produces a given photograph (Bougnoux, 1998; Brown, 1971; Gennery, 1979; Hartley and Zisserman, 2003; Faig, 1975). 


\section{Related works}

A four-step camera calibration procedure with implicit image correction was carried out by Heikkila and Silven (1997). Planebased camera calibration by Sturm and Maybank (1999) was discussed. A novel camera calibration method based on genetic algorithm carried out by Chen et al. (2008) has been given. Simplified intrinsic camera calibration and hand-eye calibration for robot vision was carried out by Malm and Heyden in 2003. Perspective Geometry based single image camera calibration technique has been discussed by Avinash and Murali in 2008. In 2006, Yanqing and Zhiyan came out with a flexible camera calibration method for computer visual $3 \mathrm{~d}$ reconstruction system.

This paper gives a brief sketch on different camera calibration techniques in section 2, followed by section 3 with adaptive camera calibration technique. Section 4 describes tracking lens distortion. The undistortion process has been described in section 5 . Section 6 suggests a procedure for quality measurement of processed images/outputs. Section 7 presents results and discussion while Section 8 is the conclusion.

\section{Camera calibration techniques}

\section{A. Photogrammetric calibration}

Photogrammetry is defined as a science of making measurements from photographs. Calibration is performed by observing an object whose geometry in 3-D space is known. The calibration object usually consists of two or three planes orthogonal to each other. Sometimes, a plane undergoing a precisely known translation is also used. These approaches require an expensive Calibration setup (Knyaz, 2006; Maas, 1997; Remondino and Borlin, 2004; Weckesser and Hetzel, 1994; Ritter et al., 2006).

\section{B. Self-calibration}

Calibration methods can be used when no Euclidean information is available, and, if properly designed, can also cope with varying intrinsic parameters. Knowledge of the camera motion and the intrinsic parameters allows for the Euclidean reconstruction of the scene. Images taken by the moving camera on a static image plane with fixed internal parameters are sufficient to recover both the internal and external parameters which allow reconstructing 3-D structure. This approach is very flexible. There are many parameters to estimate; sometime we cannot always obtain reliable results (Hartley, 1994; Luong and Faugeras, 1997; Hua et al., 2005; Maybank and Faugeras, 1992; Paulo et al., 1999).

\section{Zhengyou Zhang method}

This technique only requires the camera to observe a planar pattern shown at a few (at least two) different orientations. Zhang has developed a toolbox that allows others to implement calibrations that involve algorithms related to their personal research. This procedure is relatively accurate. In this technique several checkerboards pattern images (here 9x7 boxes) in various position with different distance are captured by a single digital camera (Zhang, 1998; Zhang, 1999).

\section{Mathematical Representation of 3-D Model Images}

A $2 \mathrm{D}$ point is denoted by $\mathrm{m}=[\mathrm{u}, \mathrm{v}]^{\mathrm{T}}$. A $3 \mathrm{D}$ point is denoted by $\mathrm{M}=[\mathrm{X}, \mathrm{Y}, \mathrm{Z}]^{\mathrm{T}}$. We use $\tilde{\mathrm{x}}$ to denote the augmented vector by

adding 1 as the last element: $\tilde{\mathrm{m}}=[\mathrm{u}, \mathrm{v}, 1]^{\mathrm{T}}$ and $\tilde{\mathrm{M}}=[\mathrm{X}, \mathrm{Y}, \mathrm{Z}, 1]^{\mathrm{T}}$

A camera is modeled by the usual pinhole: the relationship between a 3D point $\mathrm{M}$ and its image projection $\mathrm{m}$ is given by

$$
\mathrm{s} \tilde{\mathrm{m}}=\mathrm{A}[\mathrm{R} \quad \mathrm{t}] \mathrm{M}
$$

and

$$
\mathrm{A}=\left[\begin{array}{ccc}
\alpha & c & u 0 \\
0 & \beta & v 0 \\
0 & 0 & 1
\end{array}\right]
$$


where $s$ is an arbitrary scale factor; $(\mathrm{R}, \mathrm{t})$, called the extrinsic parameters, is the rotation and translation which relates the world coordinate system to the camera coordinate system; A is called the camera intrinsic matrix, and $(\mathrm{u} 0, \mathrm{v} 0)$ are the coordinates of the principal point, $\alpha$ and $\beta$ the scale factors in image $\mathrm{u}$ and $\mathrm{v}$ axes, and $\mathrm{c}$ the parameter describing the skewness of the two image axes.

\section{E. Homography between the model plane and its image:}

Without loss of generality, we assume the model plane is v on $\mathrm{Z}=0$ of the world coordinate system. Let's denote the $\boldsymbol{i}_{t h}$ column of the rotation matrix R by $r_{i}$. From (1),

We have,

$$
s\left[\begin{array}{l}
u \\
v \\
1
\end{array}\right]=\mathrm{A}\left[\begin{array}{llll}
r 1 & r 2 & r 3 & t
\end{array}\right]\left[\begin{array}{c}
X \\
Y \\
0 \\
1
\end{array}\right]=\mathrm{A}\left[\begin{array}{lll}
r 1 & r 2 & t
\end{array}\right]\left[\begin{array}{c}
X \\
Y \\
1
\end{array}\right]
$$

By abuse of notation, we still use $\mathrm{M}$ to denote a point on the model plane, but since $\mathrm{Z}$ is always equal to 0 . In turn, therefore, a model points $\mathrm{M}$ and its image ms are related by a homography $\mathrm{H}$ :

with

$$
\tilde{\mathrm{Sm}}=\mathrm{H} \tilde{\mathrm{M}}
$$

$$
\mathrm{H}=\mathrm{A}\left[\begin{array}{lll}
\mathrm{r} 1 & \mathrm{r} 2 & \mathrm{t}
\end{array}\right]
$$

The $3 \times 3$ matrix $\mathrm{H}$ is defined up to a scale factor.

\section{Solving camera calibration}

This section provides the details regarding how to solve the camera calibration problem, effectively. The straight lines in several orientations throughout these images are used to determine the pattern of radial lens distortion. Figure 2. Shows the calibration process have been adapted here.

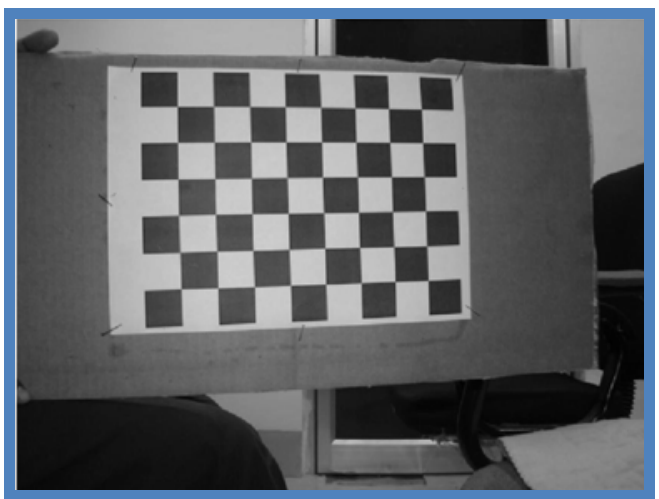

Figure 1. Original image (640pix X 480pix) of a calibration checkerboard pattern, taken with a Sony Cyber Shot DSC-H50 camera

\section{A. Determining the radial distortion coefficients}

The first part of the calibration process is to determine an image coordinate remapping that causes images taken by the camera to be true perspective images, that is, straight lines in the world project as straight lines in the image. The procedure makes use of one or several images with many known straight lines in it. Architectural scenes are usually a rich source of straight lines, but for most of the work here we used pictures of the checkerboard pattern shown below (Figure 1) to determine the radial lens distortion.

The checkerboard pattern is a natural choice since straight lines with easily localized endpoints and interior points can be found in several orientations (horizontal, vertical, and various diagonals) throughout the image plane. The checkerboard pattern also has the desirable property that its corners are localizable independent of the linearity of the image response. That is, applying a nonlinear monotonic function to the intensity values of the checkerboard image, such as gamma correction, does not affect corner 
localization. As a counter example, this is not the case for the corners of a white square on a black background. If the image is blurred somewhat, changing the image gamma will cause the square to shrink or enlarge, which will affect corner localization.

The pattern in Figure 1 was photographed on a Sony Cyber Shot DSC-H50camera. Since this lens, like most, changes its internal configuration depending on the distance it is focused at, it is possible that its pattern of radial distortion could be different depending on where it is focused. Thus, care was taken to focus the lens at infinity and to reduce the aperture until the image was adequately sharp.

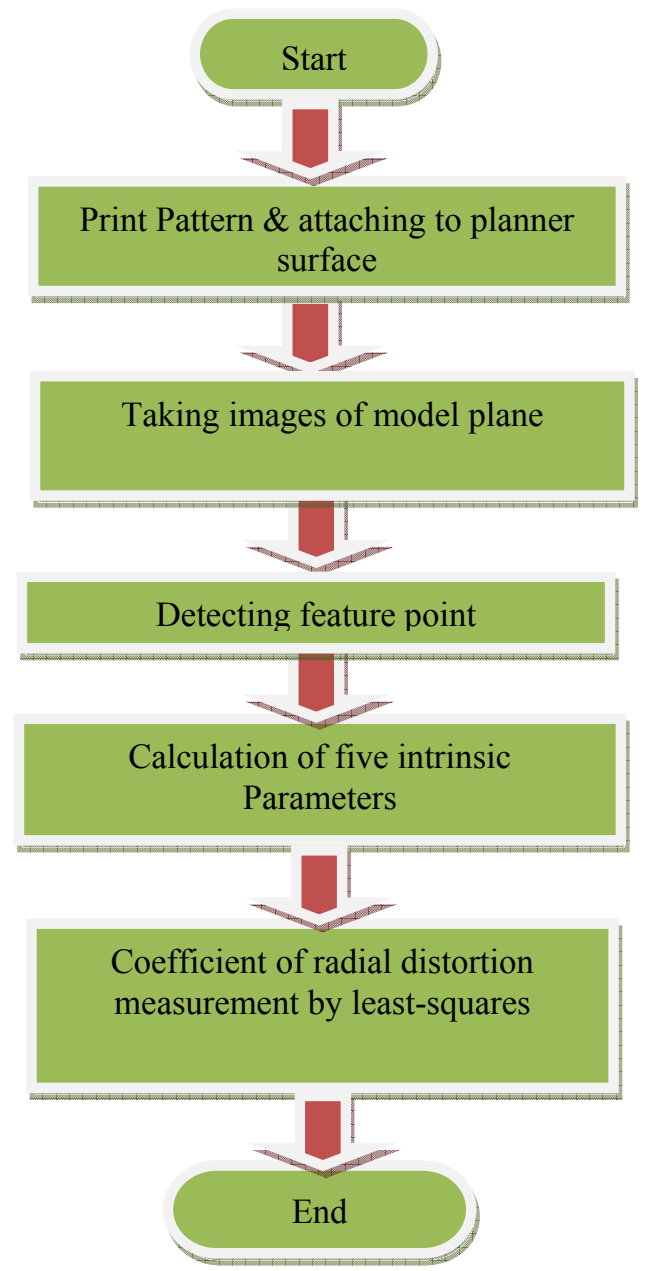

Figure 2. Flow chart for showing the calibration process

\section{Tracking lens distortion}

\section{A. Sobel edge detector}

For more easily visually tracking lens distortion of the test images, simple Sobel edge detector process is run which produce the image shown in Figure 2. Mathematically, the operator uses two $3 \times 3$ kernels which are convolved with the original image to calculate approximations of the derivatives - one for horizontal changes, and one for vertical. If we define I as the source image, and there are two images which at each point contain the horizontal and vertical derivative approximations, the computations are as follows:

$$
\mathrm{P}_{\mathrm{y}}=\left[\begin{array}{ccc}
+1 & +2 & +1 \\
0 & 0 & 0 \\
-1 & -2 & -1
\end{array}\right] * \mathrm{I}
$$




$$
\mathrm{P}_{\mathrm{x}}=\left[\begin{array}{ccc}
+1 & 0 & -1 \\
+2 & 0 & -2 \\
+1 & 0 & -1
\end{array}\right] * \mathrm{I}
$$

where * here denotes the 2-dimensional convolution operation.

The x-coordinate is here defined as increasing in the "right"-direction, and the y-coordinate is defined as increasing in the "down"-direction. At each point in the image, the resulting gradient approximations can be combined to give the gradient magnitude.

Using this information, we can also calculate the gradient's direction:

$$
\mathrm{P}=\sqrt{\left(\mathrm{P}_{\mathrm{x}}^{2}+\mathrm{P}_{\mathrm{y}}{ }^{2}\right)}
$$

Using this information, we can also calculate the gradient's direction:

$$
\Theta=\arctan \left(\frac{P_{x}}{P_{y}}\right)
$$

The pattern of distortion can now be made evident to a human observer by shrinking this edge image in either the horizontal or the vertical direction by an extreme amount.

Table 1. A 9x9 Convolution filter that detects even corners of the checkerboard pattern

\begin{tabular}{|l|l|l|l|l|l|l|l|l|}
\hline 1 & 1 & 1 & 1 & 0 & -1 & -1 & -1 & -1 \\
\hline 1 & 1 & 1 & 1 & 0 & -1 & -1 & -1 & -1 \\
\hline 1 & 1 & 1 & 1 & 0 & -1 & -1 & -1 & -1 \\
\hline 1 & 1 & 1 & 1 & 0 & -1 & -1 & -1 & -1 \\
\hline 0 & 0 & 0 & 0 & 0 & 0 & 0 & 0 & 0 \\
\hline-1 & -1 & -1 & -1 & 0 & 1 & 1 & 1 & 1 \\
\hline-1 & -1 & -1 & -1 & 0 & 1 & 1 & 1 & 1 \\
\hline-1 & -1 & -1 & -1 & 0 & 1 & 1 & 1 & 1 \\
\hline-1 & -1 & -1 & -1 & 0 & 1 & 1 & 1 & 1 \\
\hline
\end{tabular}

Table 2. A 9x9 Convolution filter that detects odd corners of the checkerboard pattern

\begin{tabular}{|l|l|l|l|l|l|l|l|l|}
\hline-1 & -1 & -1 & -1 & 0 & 1 & 1 & 1 & 1 \\
\hline-1 & -1 & -1 & -1 & 0 & 1 & 1 & 1 & 1 \\
\hline-1 & -1 & -1 & -1 & 0 & 1 & 1 & 1 & 1 \\
\hline-1 & -1 & -1 & -1 & 0 & 1 & 1 & 1 & 1 \\
\hline 0 & 0 & 0 & 0 & 0 & 0 & 0 & 0 & 0 \\
\hline 1 & 1 & 1 & 1 & 0 & -1 & -1 & -1 & -1 \\
\hline 1 & 1 & 1 & 1 & 0 & -1 & -1 & -1 & -1 \\
\hline 1 & 1 & 1 & 1 & 0 & -1 & -1 & -1 & -1 \\
\hline 1 & 1 & 1 & 1 & 0 & -1 & -1 & -1 & -1 \\
\hline
\end{tabular}

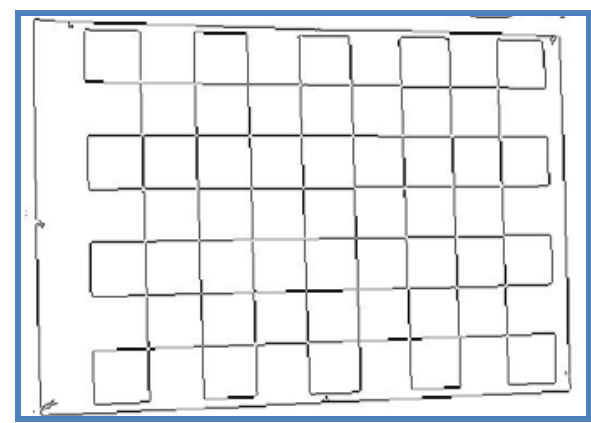

Figure 3. The edges of the checkerboard pattern Found by using Sobel edge detector of the Undistorted Pattern

We observed that lines passing through the center of the image stay straight, as do the vertical lines at the extreme left and right of the image. Lines which lie at intermediate distances from the center of the image are bowed. 
Since this filter Table-1\&2 itself resembles a checkerboard pattern, it gives a strong response (positive or negative, depending on which type of corner) when centered over a checkerboard corner. Taking the absolute value of the filter output produces an image where the checkerboard corners appear as white dots, as in Figure 4 showing the odd and even position of the corners.

Image points can be easily localized by first convolving the image with the filter in Tables $1 \& 2$. In abstract terms a convolution is defined as a product of functions and that are objects in the algebra of Schwartz function in. Convolution of two functions and over a finite range is given by

$$
[f * g](t) \equiv \int_{0} f(\tau) g(t-\tau) d \tau
$$

Where the symbol denotes convolution of and. Once the distortion parameters are solved for, it is possible to undistort any image taken with the same lens as the calibration images so that straight lines in the world image to straight lines on the image plane (Foster, 2006).

\section{Undistortion image of Braille paper}

The undistortion process of the paper can be achieved by the method described in Zhang (1998). By comparing both Figure 4 and 5 we can observe a significant lens distortion. The dimension of Braille cell can be represented by maximum six dots and is shown in Figure 7.

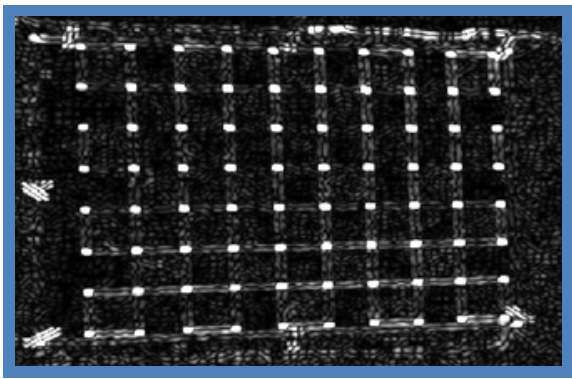

Figure 3. The results of convolving the undistorted checkerboard image with the filter in Table 2 and 3 , and taking the absolute value of the filter outputs

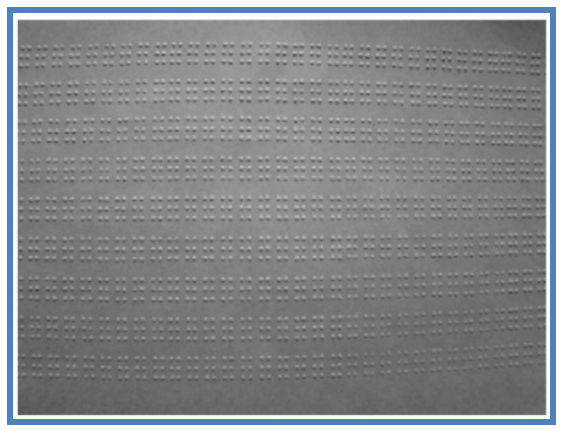

Figure 4. Braille original gray Image taken withSonyDSC-H50, showing Barrel distortion

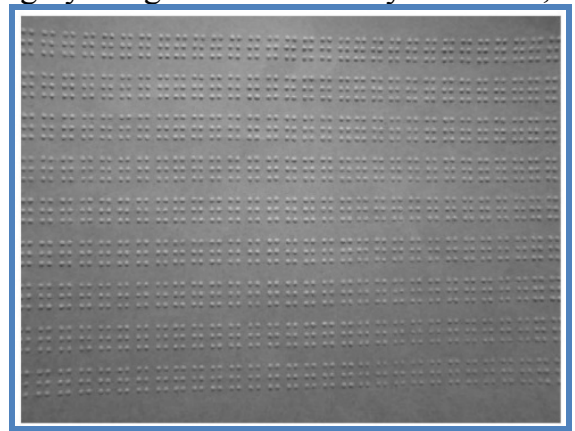

Figure 5. Undistorted Braille image 


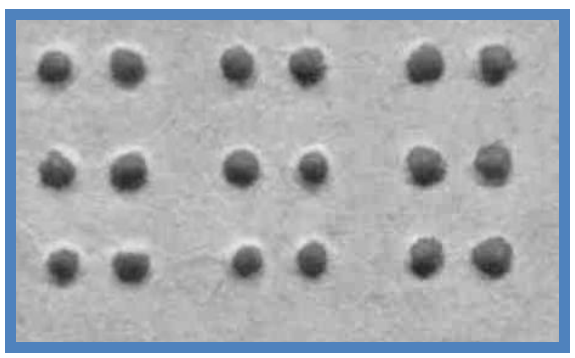

Figure 6. Gray scale image of Braille Paper with selected 18 dots

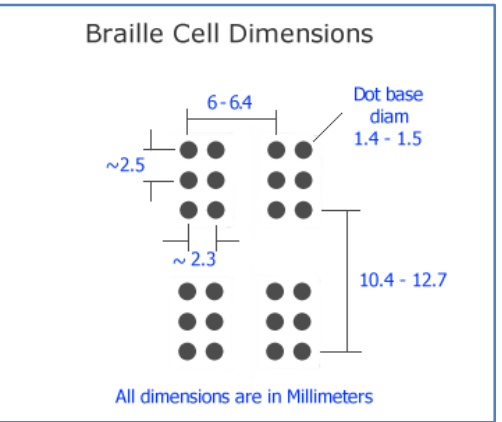

Figure 7. Braille cell dimension

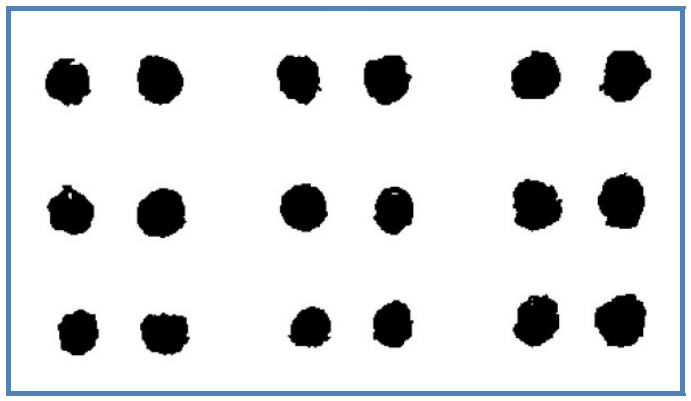

Figure 8 . Threshold image by the thresholding value $=.5451$

\section{Procedure for the quality measurement}

\section{A. Process for finding distance between dots}

Thresholding is an image processing technique for converting a grayscale or color image to a binary image based upon a threshold value. If a pixel in the image has an intensity value less than the threshold value $(\mathrm{Vth}=0.5451)$, the corresponding pixel in the resultant image is set to black. Otherwise, if the pixel intensity value is greater than or equal to the threshold intensity, the resulting pixel is set to white. Image thresholding is very useful for keeping the significant part of an image and getting rid of the unimportant part or noise. This holds true under the assumption that a reasonable threshold value is chosen. Figure 8 shows the threshold image of printed Braille paper with the value of .5451.

Removing small objects-removes from a binary image all connected components (objects) that have fewer than P pixels, producing another binary image, BW2. The default connectivity is 8 for two dimensions, 26 for three dimensions. Label connected components in a binary image returns a matrix $\mathrm{L}$ of the same size as BW.

The centroid algorithm is based on the standard center of mass equation in discrete form (McDowell, 2004).

Where:

$$
R_{c m}=\frac{\sum_{j=j \min }^{j \max } \sum_{i=i \min }^{i \max }\left[i \cdot f(j, i) \hat{u}_{x}+j \cdot f(j, i) \hat{u}_{y}\right]}{\sum_{j=j \min }^{j \max } \sum_{j=j \min }^{j \max } f(j, i)}
$$

$i_{\min }=$ minimum column index,$i_{\max }=$ maximum column index, $j_{\min }=$ minimum row index, $j_{\max }=$ maximum row index 


\section{Results and discussion}

The camera to be calibrated is Sony Cyber Shot DSC-H50 camera. The image resolution is $640 \mathrm{x} 480$. The model plane contains a pattern of 9x7 squares, so there are 252 corners. The size of the pattern is $29 \mathrm{~mm}$.Five images of the plane under different orientations were taken, as shown above. We can observe a significant lens distortion in the images. The corners were detected as the intersection of straight lines fitted to each square. Our experiments are composed of single camera calibration setup. For the single camera experiments, we have tried various numbers of images. On the other hand, we found that using more than 5 images did not increase the accuracy any more. We applied this calibration algorithm to all the 5 images. The results are shown in Table 8 .

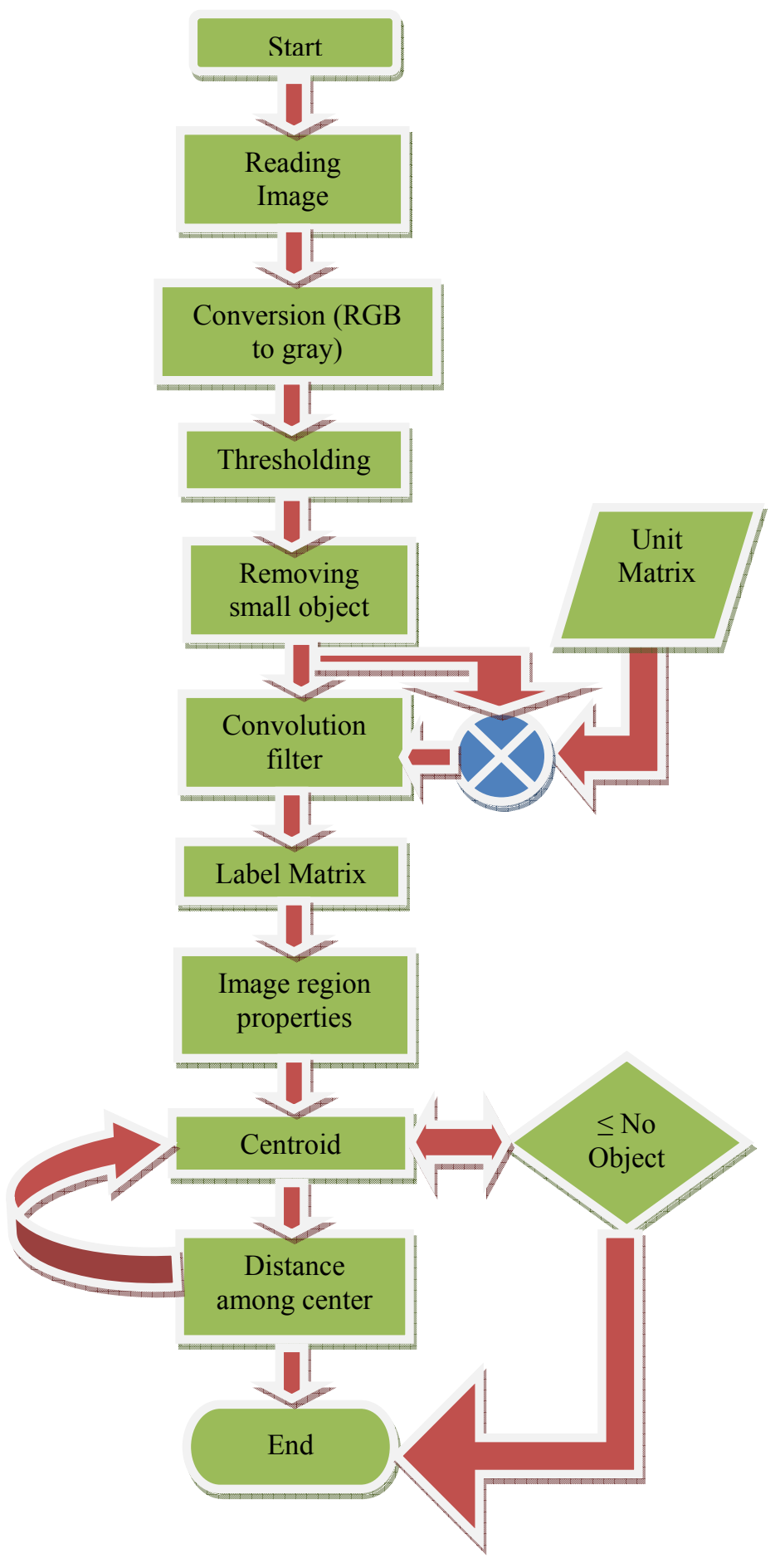

Figure 9. Showing distance measurement process 
In Table 7, we have shown the distortion in pixels against the number of images. We have taken the Corner points of the original (distorted) Image and the corner point of the undistorted images. Due to undistortion of the image the corner point will shift with respect to the original image. We can see that the distortion is varied with number of image shown in Figure 11.

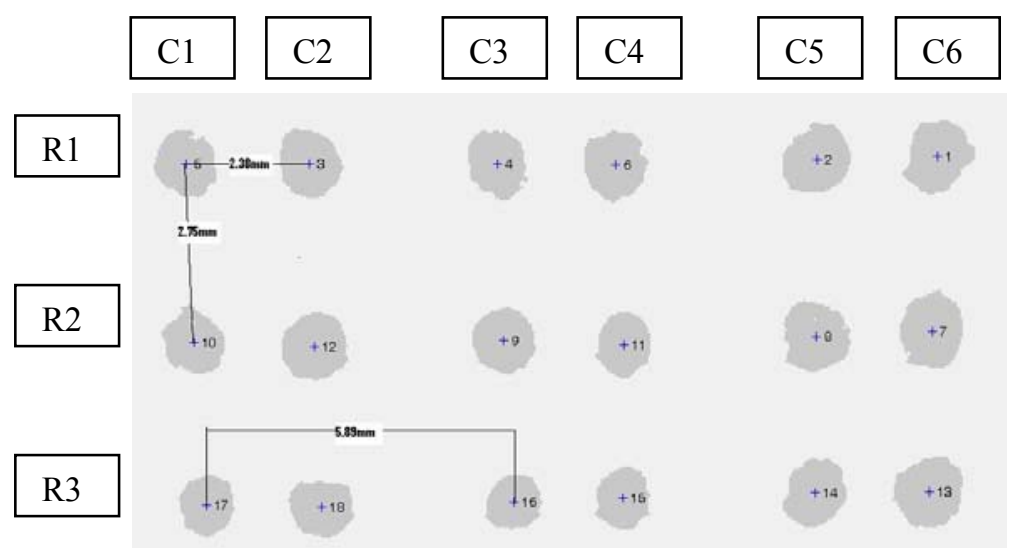

Figure 10. Detected Braille objects by centroid method Scanned from left to right

Table 3. Distance $(\mathrm{mm})$ between the centroids of R1 and R2

\begin{tabular}{|c|c|c|c|c|c|c|c|}
\hline \multicolumn{6}{|c|}{ 1R2 $(\mathrm{mm})$} & Avg. & Error(mm) \\
\hline 2.48 & 2.74 & 2.71 & 2.94 & 2.82 & 2.96 & 2.75 & $2.75 \pm 2.5= \pm .25$ \\
\hline 9.37 & 10.35 & 10.24 & 11.11 & 10.65 & 11.18 & 10.39 & $.94($ pixels) \\
\hline
\end{tabular}

Table 4. Distance ( $\mathrm{mm})$ between the centroids of $\mathrm{C} 1$ and $\mathrm{C} 3$

\begin{tabular}{|c|c|c|c|c|c|c|c|}
\hline \multicolumn{6}{|c|}{$\mathrm{C} 1 \mathrm{C} 3(\mathrm{~mm})$} & Avg. & Error \\
\hline 5.72 & 6.34 & 5.92 & 5.11 & 5.72 & 6.55 & 5.89 & $5.89 \pm 6.64= \pm .75$ \\
\hline 21.61 & 23.96 & 22.37 & 19.31 & 21.61 & 24.75 & 22.26 & 2.83(pixels) \\
\hline
\end{tabular}

Table 5. Distance $(\mathrm{mm})$ between the centroids of $\mathrm{C} 1$ and $\mathrm{C} 2$

\begin{tabular}{|c|c|c|c|c|c|c|c|}
\hline \multicolumn{6}{|c|}{$\mathrm{C} 1 \mathrm{C} 2(\mathrm{~mm})$} & Avg. & Error \\
\hline 2.66 & 2.24 & 2.45 & 2.24 & 2.65 & 2.04 & 2.38 & $2.38 \pm 2.3= \pm .08$ \\
\hline 10.05 & 8.46 & 9.25 & 8.46 & 10.01 & 7.71 & 8.99 & .30 (pixels) \\
\hline
\end{tabular}

Table 6. Distance ( $\mathrm{mm}$ ) between the centroids of R3 and R6

\begin{tabular}{|c|c|c|c|c|c|c|c|}
\hline \multicolumn{6}{|c|}{ R3R6(mm) } & Avg. & Error \\
\hline 10.97 & 11.08 & 13.18 & 10 & 9.24 & 7.9 & 10.39 & $10.39 \pm 10.4= \pm .01$ \\
\hline 41.46 & 41.87 & 49.81 & 37.79 & 34.92 & 29.85 & 39.26 & .03 (pixels) \\
\hline
\end{tabular}

Table 7. Distortion results of single camera calibration

\begin{tabular}{|c|c|}
\hline No of images & $\begin{array}{c}\text { Distortion } \\
\text { (per pixels) }\end{array}$ \\
\hline 1 & 1.450092 \\
\hline 2 & 1.553057 \\
\hline 3 & 1.783507 \\
\hline 4 & 1.462205 \\
\hline 5 & 1.62168 \\
\hline
\end{tabular}


Table 8 . Result of calibration processed by Matlab

\begin{tabular}{|c|c|c|c|c|c|c|c|c|c|c|c|}
\hline \multirow{2}{*}{$\begin{array}{l}\text { No Of } \\
\text { Image }\end{array}$} & \multirow{2}{*}{\multicolumn{2}{|c|}{$\begin{array}{c}\text { Focal } \\
\text { Length(fc) }\end{array}$}} & \multirow{2}{*}{\multicolumn{2}{|c|}{$\begin{array}{l}\text { Principal } \\
\text { Point(cc) }\end{array}$}} & \multicolumn{5}{|c|}{ Distortion $(\mathrm{kc})$} & \multirow{2}{*}{\multicolumn{2}{|c|}{$\begin{array}{c}\text { Pixel } \\
\text { Error(err) }\end{array}$}} \\
\hline & & & & & \multirow{3}{*}{$\begin{array}{c}\mathrm{K} 1 \\
- \\
0.14421\end{array}$} & \multirow{3}{*}{$\begin{array}{c}\mathrm{K} 2 \\
- \\
0.06549\end{array}$} & \multirow{3}{*}{$\begin{array}{c}\mathrm{K} 3 \\
0.00581\end{array}$} & \multirow{3}{*}{$\begin{array}{c}\mathrm{K} 4 \\
0.011 \\
79\end{array}$} & \multirow{3}{*}{$\begin{array}{c}\mathrm{K} 5 \\
0.00 \\
000\end{array}$} & & \\
\hline 5 & 801.1 & 793.7 & 312.0 & 222.2 & & & & & & 0.444 & 0.387 \\
\hline & 3189 & 016 & 7083 & 7948 & & & & & & 51 & 17 \\
\hline 4 & $\begin{array}{l}693.5 \\
3180\end{array}$ & $\begin{array}{l}692.0 \\
4824\end{array}$ & $\begin{array}{l}319.5 \\
0000\end{array}$ & $\begin{array}{c}239.5 \\
0000\end{array}$ & $\begin{array}{c}- \\
0.22984\end{array}$ & 0.26423 & $\begin{array}{c}- \\
0.00194\end{array}$ & $\begin{array}{c}-0.00 \\
043\end{array}$ & $\begin{array}{c}0.00 \\
000\end{array}$ & $\begin{array}{c}0.294 \\
52\end{array}$ & $\begin{array}{c}0.395 \\
65\end{array}$ \\
\hline 3 & $\begin{array}{l}633.5 \\
0177\end{array}$ & $\begin{array}{l}627.9 \\
2847\end{array}$ & $\begin{array}{l}293.2 \\
6251\end{array}$ & $\begin{array}{c}236.5 \\
6528\end{array}$ & $\begin{array}{c}- \\
0.21249\end{array}$ & 0.30255 & $\begin{array}{c}- \\
0.00360\end{array}$ & $\begin{array}{c}0.008 \\
24\end{array}$ & $\begin{array}{l}0.00 \\
000\end{array}$ & $\begin{array}{c}0.307 \\
90\end{array}$ & $\begin{array}{c}0.364 \\
92\end{array}$ \\
\hline 2 & $\begin{array}{l}613.6 \\
9139\end{array}$ & $\begin{array}{l}612.2 \\
6030\end{array}$ & $\begin{array}{l}319.5 \\
0000\end{array}$ & $\begin{array}{c}239.5 \\
0000\end{array}$ & $\begin{array}{c}- \\
0.18865\end{array}$ & 0.13955 & $\begin{array}{c}- \\
0.00609\end{array}$ & $\begin{array}{c}0.000 \\
59\end{array}$ & $\begin{array}{c}0.00 \\
000\end{array}$ & $\begin{array}{c}0.284 \\
22\end{array}$ & $\begin{array}{c}0.292 \\
31\end{array}$ \\
\hline 1 & $\begin{array}{c}567.4 \\
4421\end{array}$ & $\begin{array}{l}567.1 \\
9535\end{array}$ & $\begin{array}{l}319.5 \\
0000\end{array}$ & $\begin{array}{c}239.5 \\
0000\end{array}$ & $\begin{array}{c}- \\
0.15842\end{array}$ & 0.11923 & $\begin{array}{c}- \\
0.00457\end{array}$ & $\begin{array}{c}0.003 \\
12\end{array}$ & $\begin{array}{c}0.00 \\
000\end{array}$ & $\begin{array}{c}0.276 \\
69\end{array}$ & $\begin{array}{c}0.294 \\
26\end{array}$ \\
\hline
\end{tabular}

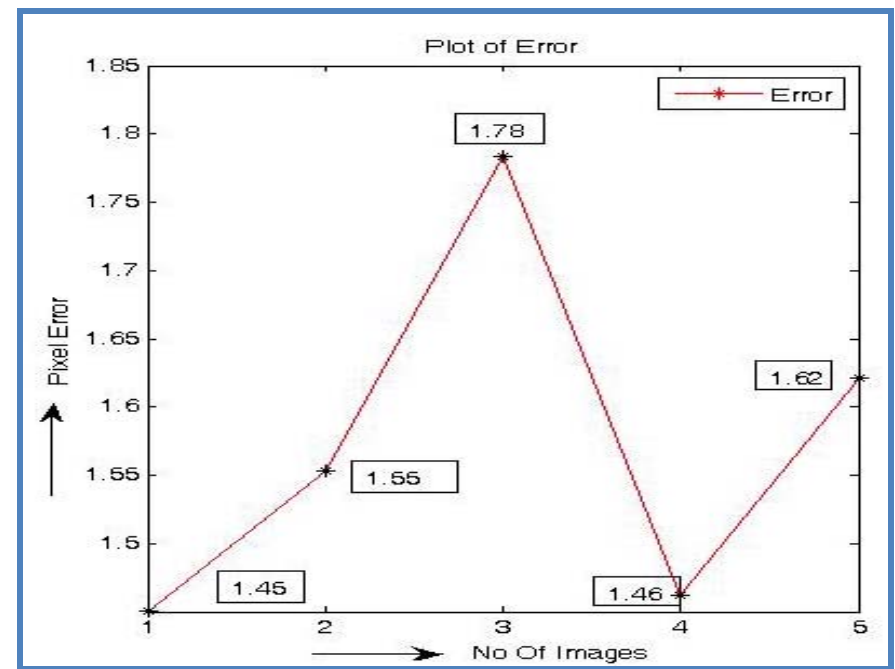

Figure 11. Average Pixel error of each corner point of the model plane

\section{Conclusion}

An advantage of research work for camera calibration is that this method is much closer to accuracy than other processes used for object detection by centroid measurement and distance calculation. Zhengyou Zhang Method for camera calibration has been followed. This technique only requires the camera to observe a planar pattern shown at a few (at least two) different orientations. This research work has been carried out at Central Mechanical Engineering Research Institute, Durgapur, India and mainly focused on finding equal distance in the Braille printer output by image processing technique. The distorted images are compared with the modified undistorted one. Results, thereafter, finally establish this to be a very satisfactory quality testing procedure of a commercially fabricated Braille printer.

\section{Acknowledgement}

The authors are grateful to Director, CMERI, Durgapur for extending his help regarding this project work. We thank Somajyoti Majumder scientist CMERI, Durgapur for his technical help, good ideas and valuable assistance during the experiment.

\section{References}

Avinash N. and Murali S., 2008. Perspective geometry based single image camera calibration. Journal of Mathematical Imaging and Vision, Springer Netherlands, Vol 30 , No. 3, pp 221 - 230.

Bougnoux S., 1998. From projective to euclidean space under any practical situation, a criticism of self-calibration. 6th International Conference on Computer Vision, Bombay, India, pp. 790-796.

Brown D. C., 1971. Close-range camera calibration. Photogrammetric Engineering , pp. 855-866. 
Chen F, Zhao J. and Zhao H.W., 2008. A novel camera calibration method based on genetic algorithm. IEEE Transactions, Singapore, pp. 2222-2227.

Faig W., 1975. Calibration of close-range photogrammetry systems: Mathematical formulation. Photogrammetric Engineering and Remote Sensing, Vol. 41, No. 12, pp. 1479-1486.

Foster M. P., 2006.Normalized Convolution Techniques. MP Foster - clara.net.

Gennery D., 1979. Stereo-camera calibration. In Proc. $10^{\text {th }}$ Image Understanding Workshop, Stanford University Stanford, California 94305, pp. 101-108.

Hartley R. and Zisserman A., 2003 . Multiple View Geometry in Computer Vision. Cambridge University Press, second edition, ISBN 0521540518 , pp. 155-157.

Hartley R.I., 1994.An algorithm for self calibration from several views. IEEE Conference on Computer Vision and Pattern Recognition, pp. 908-912.

Hentzshel T.W., 1993. An optical braille reading system. M.Sc. Thesis, University of Manchester Institute of Science and Technology. United Kingdom.

Heikkila J. and Silven O. 1997. A four-step camera calibration procedure with implicit image correction. InfoTech Oulu and Department of Electrical Engineering University of Oulu FIN-90570 Oulu, Finland.

Hua T.P., Sugiyama A. and Faucon G. 2005. A new self-calibration technique for adaptive microphone arrays, 2005 International Workshop on Acoustic Echo and Noise Control High Tech Campus, Eindhoven, The Netherlands, September 12 - 15, 2005, pp. 237-240.

Knyaz V.A., 2006. Automated calibration technique for photogrammetric system based on a multi-media projector and a CCD camera, ISPRS Image Engineering and Vision Metrology, Dresden, pp. 25-27.

Luong Q.T.and Faugeras O., 1997.Self-calibration of a Table IX technical data of the imaging system (Sony DSC-H50) moving camera from point correspondences and fundamental matrices. International Journal of Computer Vision, Vol. 22, No. 3, pp. 261-289.

Maas H. G., 1997. Dynamic photogrammetric calibration of industrial robots. in videometrics V, SPIE Proceedings Series, Vol. 3174, SPIE's 42nd Annual Meeting, San Diego, 27.7.- 1.8.

Malm H. and Heyden A., 2003. Simplified intrinsic camera calibration and hand-eye calibration for robot vision. IEEE Conference on Intelligent Robots and Systems, Las Vegas, Nevada.

Maybank S.J. and Faugeras O.D., 1992 .A theory of self calibration of a moving camera. International Journal of Computer Vision, Vol. 8, No. 2, pp.123-152.

McDowell M., 2004. An integrated centroid finding and particle overlap decomposition algorithm for stereo imaging velocimeter. NASA Technical Memorandum (NASA/TM-2004-213365).

Paulo R. S., Mendonc A. and Cipolla R., 1999. A simple technique for self-calibration. Conference on Computer Vision and Pattern Recognition (CVPR '99), 23-25 June 1999, Ft. Collins, CO, USA. IEEE Computer Society, ISBN 0-7695-0149-4, pp. 1063-6919.

Remondino F. and Borlin N. 2004. Photogrammetric calibration of image sequences acquired with a rotating camera. Int. Archives of Photogrammetry and Remote Sensing, Vol.34 (5/W16).

Ritter M., Hemmleb. M., Faber P.C., Lich B. and Hohenberg H. 2006. Sem/fib stage calibration with photogrammetric methods. ISPRS Commission V Symp. 2006 (Int. Archives of Photogrammetry, Remote Sensing and Spatial Information Sciences), Vol. XXXVI Part 5.

Salvi J., Armangu X. and Batlle J., 2002. A comparative review of camera calibrating methods with accuracy evaluation. Pattern Recognition, pp. 1617-1635.

Sturm P. F and Maybank S. J., 1999. On plane-based camera calibration: a general algorithm, singularities, applications. Computational Vision Group, Department of Computer Science, The University of Reading Whiteknights, IEEE 1063-6919/99.

Weckesser P. and Hetzel G., 1994. photogrammetric calibration method for an active stereo vision system. Intelligent Robotic Systems (IRS), pp. 430-436.

Yanqing Z. and Zhiyan W., 2006. A flexible camera calibration method for computer visual 3D reconstruction system. IEEE Transactions 0-7803-9737-1/06, Vol 2, Guilin, China.

Zhang Z.. A flexible new technique for camera calibration. Technical Report MSRTR-98-71, Microsoft Research, December 1998. Available together with the software at http://research.microsoft.com/ zhang/Calib/

Zhang Z., 1999.Flexible Camera Calibration By Viewing a Plane From Unknown Orientations. International Conference on Computer Vision, Corfu, Greece, pp. 666-673.

\section{Biographical notes}

Suman Karmakar received his bachelor's degree in Computer Science and Engineering Department from Bankura Unnayani Institute of Engineering, Bankura under West Bengal University of Technology in 2007. He joined Central Mechanical Engineering Research Institute, Durgapur as a Project Fellow in 2007. He involved in different project like "Development of Software for the Optimization of Silicon Rubber 
Mould Characteristics", and "Development of high speed Braille Printer". His work area includes programming in VC++ and image processing using Matlab and $\mathrm{C}++$.

Rudra Prasad Chatterjee (M'08) became a Member (M) of IEEE in 2008 of Communication Society. He received his bachelor's degree in Electronics and Communication Engineering from Dr.B.C. Roy Engineering College, Durgapur under West Bengal University of Technology in 2005. He joined National Institute of Science and Technology, Berhampura, Orissa as a lecturer in 2005. Later he joined Central Mechanical Engineering Research Institute, Durgapur as a Scientist in 2006. His work area includes wireless control of robotic devices, mobile network etc. He has been involved in SUPRA Institutional Project (SIP-24) - "Design and Development of Snake Robot for Disaster Management". He has published ten papers including national and international journals.

Uma Datta received her bachelor's degree in Radio Physics and Electronics from Calcutta University in 1981 and Masters in Electrical from Regional Engineering College, Durgapur in 1994. She joined Central Mechanical Engineering Research Institute, Durgapur in 1984, as a Scientist. She became the Head of the Department of Electronics and Control Lab in the year of 2005. Her work area includes Instrumentation Engineering and Control System. She has supervised the following projects at CMERI: "Development of Expander Extruder for processing oil seeds with Automatic Control" (sponsored), "Design and Development of CTC roller sharpening machine with automatic control" (sponsored by: Department of Science and Technology, Govt. of India.), "Design and development of Electro-magnetic Stirrer for 1.3 Kg. Molten AL-356" (sponsored by: NMRL, Govt. of India) etc. She has published twenty papers including national and international journals.

Received June 2009

Accepted August 2009

Final acceptance in revised form September 2009

Table 9. Technical data of the imaging system (Sony DSC-H50)

\begin{tabular}{|l|l|l|l|l|l|l|}
\hline $\begin{array}{l}\text { Max } \\
\text { resolution }\end{array}$ & Image ratio w:h & Effective pixels & Sensor size & Sensor type & $\begin{array}{l}\text { Normal focus } \\
\text { range }\end{array}$ & $\begin{array}{l}\text { Aperture } \\
\text { range }\end{array}$ \\
\hline $3456 \times 2592$ & $4: 3,3: 2$ & 9.1 million & $\begin{array}{l}1 / 2.3 " \\
6.16 \times 4.62 \\
\mathrm{~mm}, 0.28 \mathrm{~cm}^{2}\end{array}$ & CCD & $50 \mathrm{~cm}$ & F2.7 - F4.5 \\
& & & & & \\
\hline
\end{tabular}

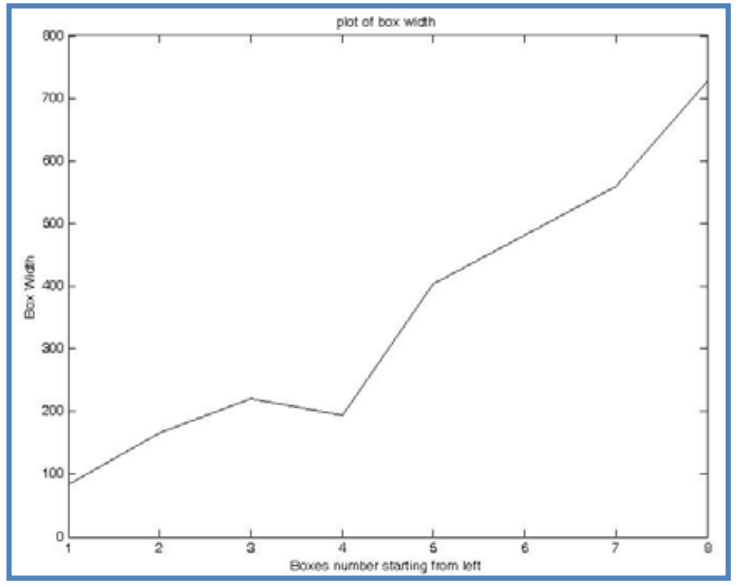

(a)

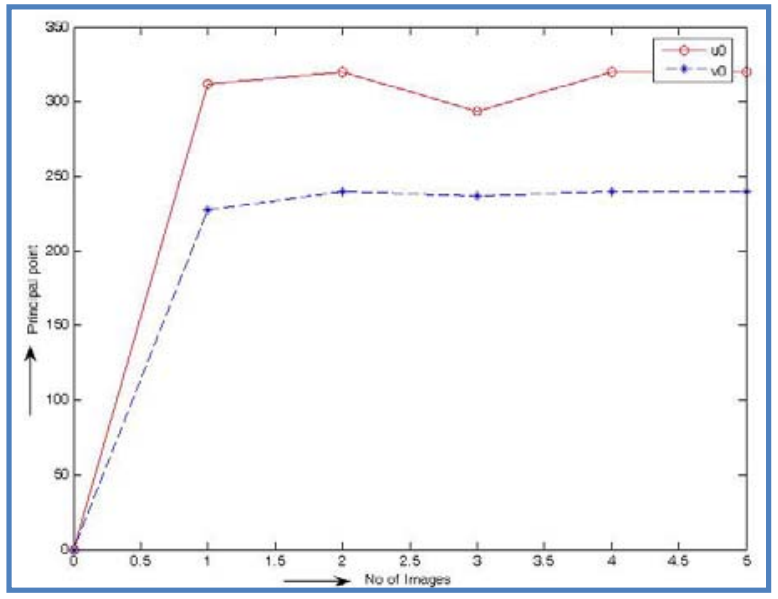

(b)

Figure 12. (a) Principal Point of the number for square widths of images of the model plane Check board squares counted from left to right (b) Note that the center of the image is around box no 5 . 


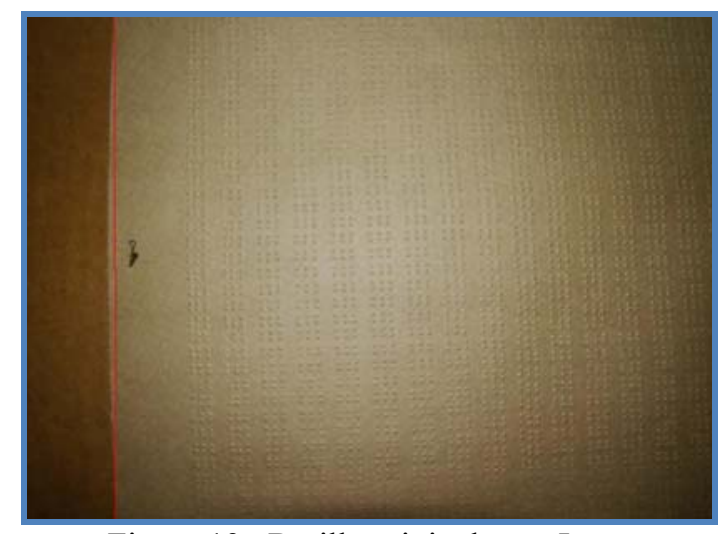

Figure 13. Braille original gray Image

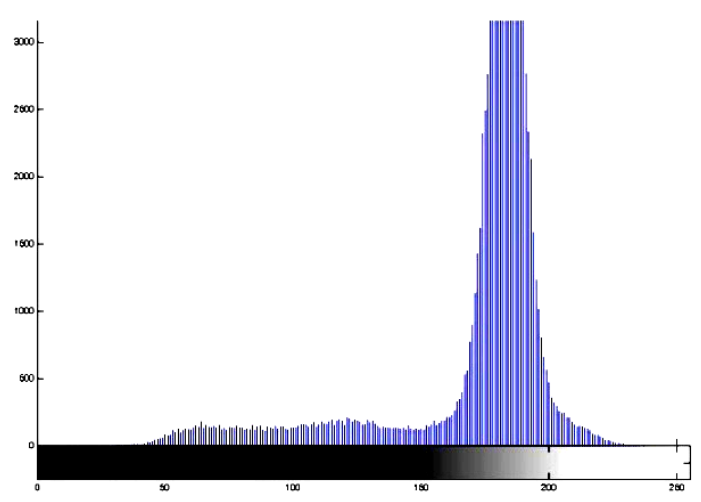

Figure 15. Histogram of the gray Braille image

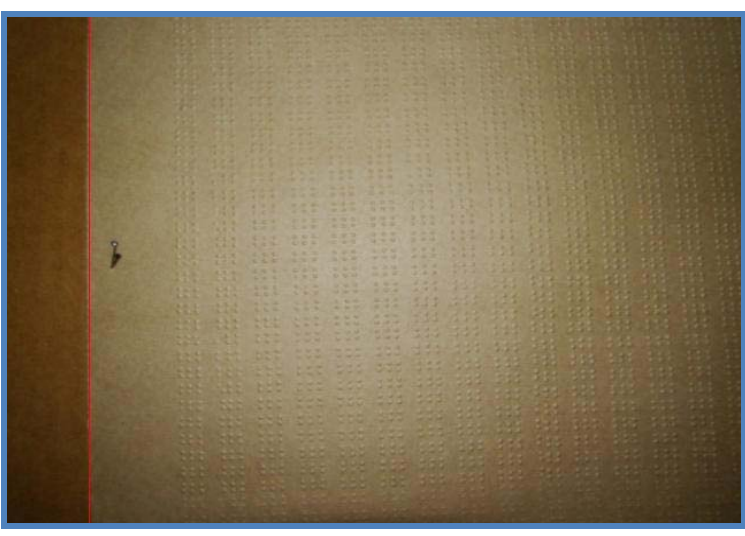

Figure 14. Braille Undistorted image

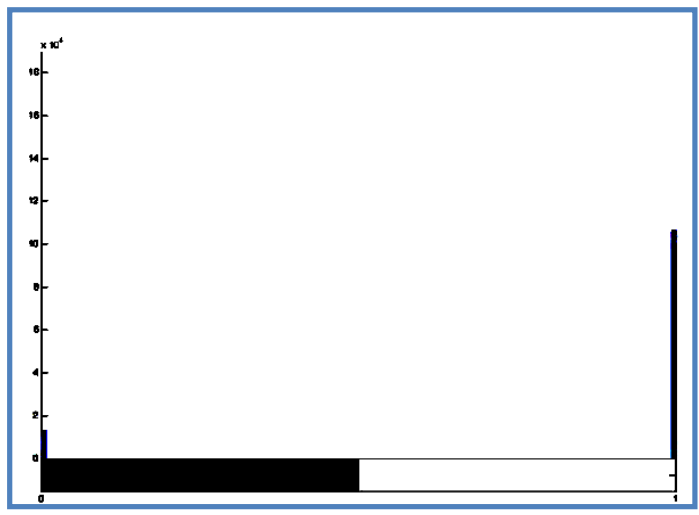

Figure 16. Histogram of the Braille threshold

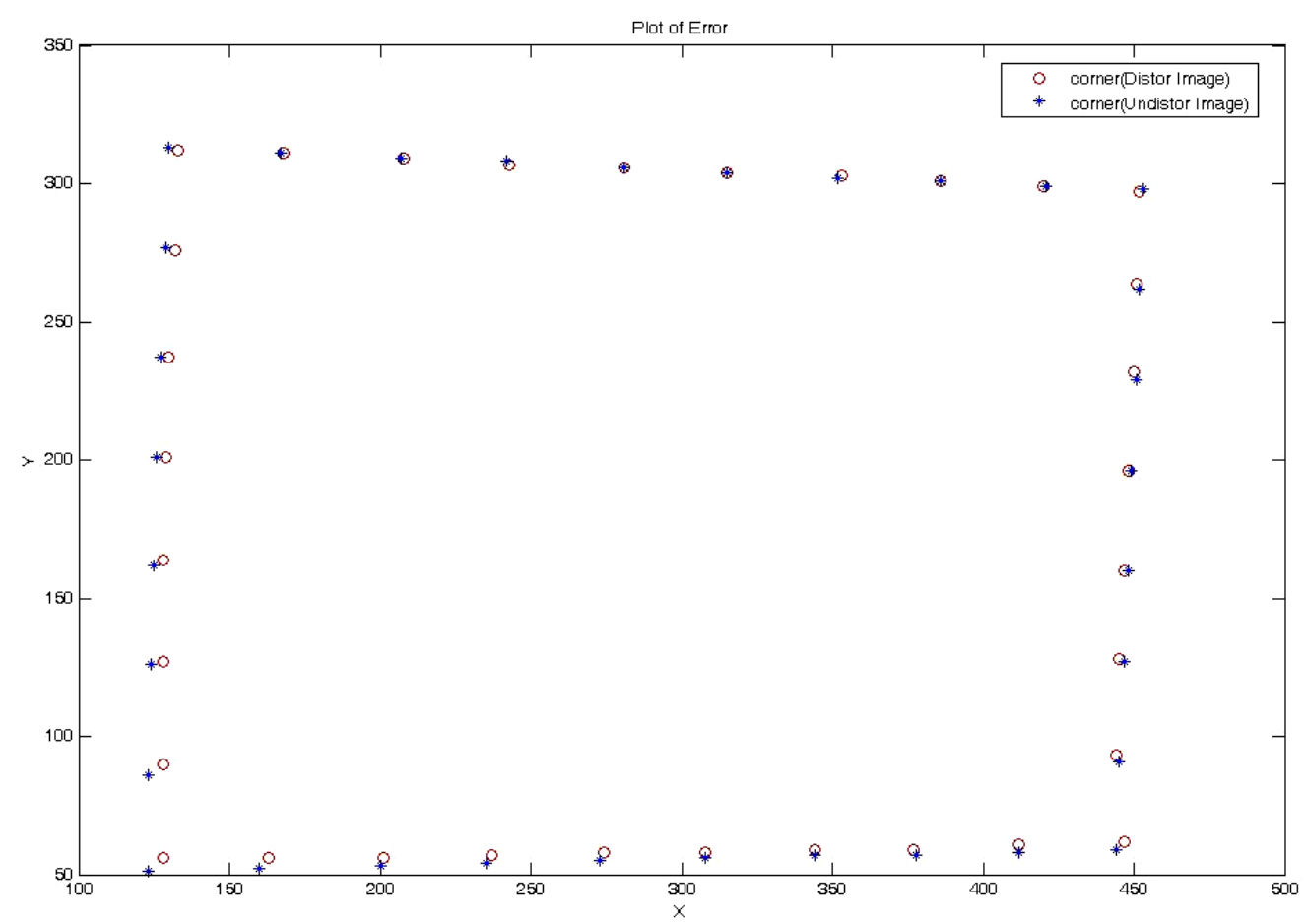

Figure 17. Shifting the corner point of undistorted model plane 

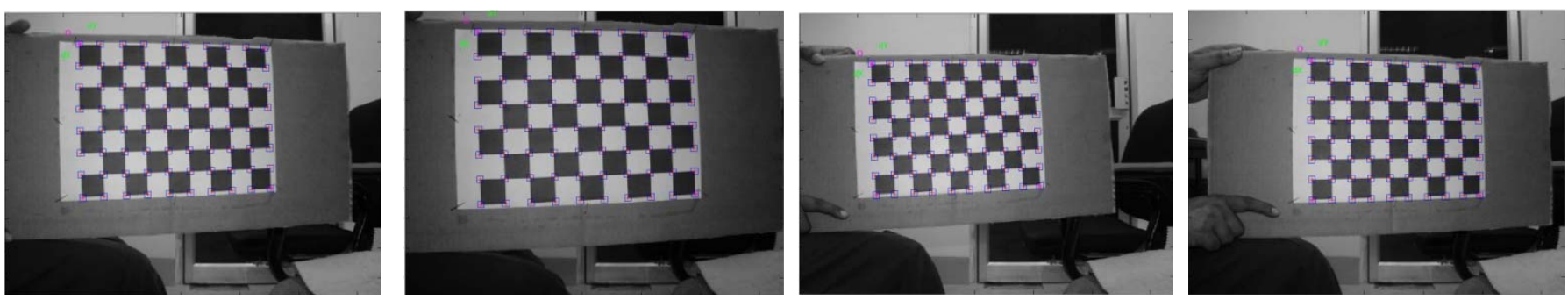

Figure 18. Five images of a model plane, together with the extracted corners (indicated by cross)
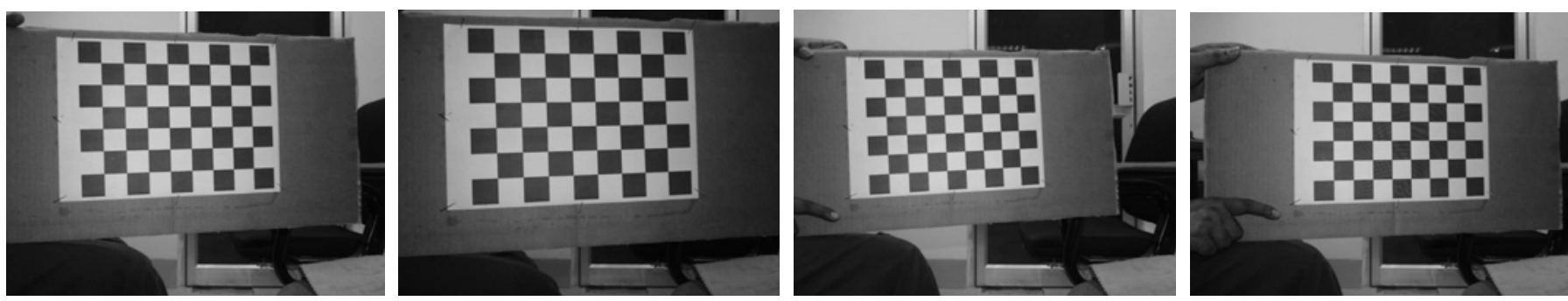

Figure 19. Five undistorted images of a model plane 